Gdańsk 2020, Nr. 43

https://doi.org/10.26881/sgg.2020.43.02

Heinz-Helmut Lüger

Universität Koblenz-Landau

\title{
Realitätsflucht und argumentativer Widerstand Kontroversen zur deutschen Ostpolitik in den 1970er Jahren
}

\begin{abstract}
Auch aus heutiger Sicht dürfte die Neuorientierung der deutschen Außenpolitik zu Beginn der 1970er Jahre kaum an Bedeutung eingebüßt haben: Unter dem Motto „Wandel durch Annäherung“ versuchte die von Willy Brandt und Walter Scheel geführte Bundesregierung, eine Verständigung mit den osteuropäischen Nachbarn auf den Weg zu bringen. Allerdings stieß diese Initiative nicht zuletzt innenpolitisch auf teilweise erbitterten Widerstand. Anhand verschiedener Dokumente soll Einblick gegeben werden in die Art der Kontroversen und den Argumentationshaushalt der Zeit.
\end{abstract}

Schlïsselwörter: Verständigung, politischer Sprachgebrauch, Argumentation, Polemik, rhetorische Mittel

Flight from reality and argumentative resistance. Ostpolitik controversy in the 1970s. Even from today's perspective, the reorientation of German foreign policy at the beginning of the 1970s didn't apparently lose its significance: With the proclamation of "change through rapprochement" policy; the German government led by Willy Brandt and Walter Scheel attempted to initiate an understanding with its Eastern European neighbours. However, this initiative aroused, especially on the domestic front, partially bitter resistance. In this paper I'd like to offer an insight into the nature of the controversy and the argumentation scope of the era on the basis of various documents.

Keywords: comprehension, political speech, argumentation, polemics, rhetorical devices

\section{Politischer Sprachgebrauch als Herausforderung}

Die Analyse politischer Texte gehört seit langem zum Gegenstandsbereich der Sprachwissenschaft, und das nicht erst, seitdem der Begriff, Politolinguistik' in Umlauf gebracht wurde. Ohne dies hier weiter vertiefen zu wollen, seien stellvertretend nur einige einschlägige Arbeiten genannt, die schon sehr früh auf wesentliche Probleme der politischen Kommunikation aufmerksam gemacht haben: Erwähnt sei zunächst der Anti-Chamberlain von Leo Spitzer (1918), eine Schrift, in der die These zurückgewiesen wird, man könne allein schon aufgrund des lexikalischen Bestands einer Sprache auf pazifistische oder kriegerische Tendenzen in einem Volk schließen. ${ }^{1}$ Mit Auswüchsen nationalsozialistischen

1 Um nur ein Beispiel anzugeben: Bei Chamberlain (1914) findet sich u. a. die Behauptung, das deutsche Lexem Friede verbinde sich wegen seiner Etymologie mit Freiheit und Freude und belege damit einen Hang 
Sprachgebrauchs beschäftigen sich später Victor KLEMPERER (1947) und Dolf STERNBERGER / Gerhard STORZ / Wilhelm SüSKIND (1957); ihre Bücher erfahren gerade in der Nachkriegszeit viel Beachtung. Im Mittelpunkt steht dabei zum einen der Einsatz von Schlagwörtern, Slogans, Kürzeln, Euphemismen, Metaphern; zum andern geht es auch um die Frage, inwieweit sich mit dem Sprachgebrauch bestimmte Beeinflussungs- oder propagandistische Manipulationsmöglichkeiten verbinden. ${ }^{2}$

Die meisten Analysen zeichnet seinerzeit eine starke Konzentration auf lexikalische Phänomene aus. Einen gewissen Höhepunkt bilden in 1970er Jahren Auseinandersetzungen um „die richtige“ Bezeichnung politischer Sachverhalte. Titel wie „Despotie der Wörter“, "Geschichte als Sprachkampf “, „Rote Semantik“ sind an der Tageordnung; die Rede ist von einem „geistigen Mauerbau“, und es herrscht gleichsam ein „Kampf um politische Begriffe“ (vgl. z. B. Kaltenbrunner 1975, Fetscher / Richter 1976).

Mit dem Aufkommen textlinguistischer und pragmatischer Fragestellungen verschieben sich nach und nach die Schwerpunkte, auch stehen methodische und theoretische Überlegungen stärker im Zentrum des Interesses. ${ }^{3}$ Gegenstand der Betrachtung sind nicht mehr einzelne sprachliche Komponenten, sondern Texte und Textmuster als ganze. In den Blick kommen Argumentationsstrukturen, akzeptanzwerbende, persuasive Strategien, die Verwendung typischer Sprachhandlungen, die Vielschichtigkeit von Textbildung und Nebenbei-Kommunikation, die Funktion von Modalitätswechsel und Mehrfachadressierung, Verfahren der Emotionalisierung, der Pejorisierung, des Polarisierens, Personalisierens und Skandalisierens sowie Mittel und Techniken, die der Selbstprofilierung, der Förderung von Gruppenidentität oder der Schaffung von Unterhaltungseffekten dienen. So gesehen, verwundert nicht, wenn die Arbeitsfelder von neueren politolinguistischen Büchern, Sammelbänden und Aufsätzen ein Bild extremer fachlicher Breite und fortschreitender Differenziertheit vermitteln. ${ }^{4}$

zu Menschlichkeit, Gerechtigkeit und Friedfertigkeit. Im Unterschied dazu würden die von lat. pax abgeleiteten Ausdrücke peace, paix, pace eher negative Vorstellungen auslösen, auf rechtliche Auseinandersetzungen, auf Pakte verweisen und letztlich für ein Fehlen moralischer Grundlagen stehen.

2 Vor einer vereinfachenden und vorschnellen Einschätzung der tatsächlichen Wirkung sprachlicher Ausdrucksformen warnen z. B. Reich (1968), Dieckmann (1969). Vgl. auch NieHR, der noch einmal mit Bezug auf vergleichbare Untersuchungen die „Macht der Wörter“ relativiert und klarstellt, man könne „den Vokabeln als solchen keine Attribute wie unmenschlich oder lügnerisch bescheinigen“"(2014: 25).

3 Aus der großen Fülle entsprechender Arbeiten sei stellvertretend verwiesen auf: BACHeM (1979), Volmert (1989), Holly (1990), Girndt (2002), MikoŁajczyk (2004), Makowski (2013), Niehr (2014). - Die hier nur stark geraffte Skizzierung von Beschreibungsansätzen ist nicht im strikten Sinn eines zeitlichen Nacheinanders zu verstehen; pragmatische Herangehensweisen finden sich auch parallel zur wortsemantisch dominierten Phase. Strenggenommen wären an dieser Stelle ebenfalls Vorarbeiten aus dem Bereich der Rhetorik einzubeziehen (vgl. KiENPOINTNER 2017).

4 Zur Veranschaulichung nur folgende Beispiele: Girnth / Spiess (2006), MaKowsKi (2011), HoLLy (2012), Olszewska / KątNy (2012), Czachur (2015), Lüger (2015), KaCZmarek (2016), KlinKer / SCharloth / SzczęK (2018), Massud (2019). 


\section{Ostpolitik im politischen Kontext}

In den folgenden Abschnitten soll es nun vor allem um die Betrachtung eines bestimmten politischen Sachverhalts aus den 1970er Jahren gehen: nämlich der neuen Ostpolitik, die die von Willy Brandt und Walter Scheel geführte bundesdeutsche Regierung unter dem Motto „Wandel durch Annäherung“ durchzusetzen versucht. Die Widerstände gegen diese Neuorientierung sind massiv und sorgen für erhebliche Spannungen zwischen den im deutschen Bundestag vertretenen Parteien. Eigentliches Ziel der Ostpolitik ist es, eine außenpolitische Neuorientierung zu erreichen, eine Verständigung mit den osteuropäischen Nachbarländern, einschließlich der DDR. Gerade aus heutiger Sicht erscheint dieser Politikwechsel - Überlegungen dazu gab es, vor allem unter Mitwirkung von Egon Bahr, schon etliche Jahre zuvor - mutig und vorausschauend:

„Was Willy Brandt und seine Mitarbeiter sich schon in den Fünfzigerjahren mitten im Kalten Krieg ausgedacht und dann in den Sechzigerjahren formuliert und umzusetzen begonnen haben, das war wohldurchdacht. Eine strategische Leistung der besonderen Art. Das gilt insbesondere für den Grundgedanken, der in die Formel, Wandel durch Annäherung'verpackt war“ (Müller 2018: 126).

Neben einem gegenseitigen Gewaltverzicht kommt besonders der Bestätigung des territorialen Status quo und damit der Respektierung der aktuellen Grenzen eine große Bedeutung zu. Bereits hieran entzünden sich heftigste Kontroversen. Gerade das Nicht-mehr in-Frage-Stellen der Westgrenze Polens, der sog. Oder-Neiße-Linie, stößt auf erbitterten Widerstand und wird gerade von der christdemokratischen Opposition schlicht als Ausverkauf deutscher Interessen gebrandmarkt. Erhellend ist in dieser Hinsicht eine Bundestags-Debatte, wie sie bereits am 22. September 1949, also einige Monate nach Gründung der Bundesrepublik, stattfindet. Zitiert seien folgende Redeausschnitte':

(1) Reimann (KPD): [...] Wir wollen in Frieden und Freundschaft mit allen Völkern leben und besonders mit den Völkern des Ostens und Südostens.

(Händeklatschen bei der KPD. - Zuruf aus der Mitte: Ihr habt die Heimat von 12 Millionen Menschen vergeben!)

Gerade die Revision stört nicht nur unser Verhältnis gegenüber Polen, sondern bedeutet in der endgültigen Konsequenz Krieg!

(Abg. Renner: Sehr gut!)

Das darf nicht sein! Unser Volk darf nicht in einem dritten Weltkrieg vernichtet werden.

(Lebhafte Zurufe.)

$\rightarrow \quad$ Die Oder-Neiße Grenze ist die Grenze des Friedens .

(Andauernde erregte Rufe: Pfui! Pfui! - Lärm. - Glocke des Präsidenten. - Erregte Zurufe: Abtreten! Abtreten!)

- Ich trete hier nicht ab, bis ich nicht alles gesagt habe!

(Fortgesetzter Lärm. - Glocke des Präsidenten.)

5 Deutscher Bundestag, 1. Wahlperiode, 7. Sitzung, 22.9.1949, S. 66; Redeprotokoll einsehbar unter: http://dip21.bundestag.de/dip21/btp/01/01007.pdf(10.10.2019). 
Präsident Dr. Köhler: Herr Abgeordneter Reimann, ich habe seit gestern - -

(Andauernde große Unruhe und Rufe: Pfui! Raus! - Abg. Strauß: Schickt ihn nach Moskau! Ziehen Sie die Uniform an! - Abg. Reimann: Ich werde hier nicht gehen!)

- Meine Damen und Herren!

(Andauernde große Unruhe. - Zurufe: Moskauer Agent! Bezahlter Provokateur! - Abg. Reimann: Das sind Sie! - Lärm.)

- Herr Abgeordneter Reimann, Sie haben eben ausgesprochen, daß die Oder-Neiße-Linie die Friedenslinie ist.

(Anhaltende große Unruhe.)

Seitdem gestern hier sämtliche Parteien gesprochen haben, haben sie übereinstimmend die Oder-Neiße-Linie als die deutsche Grenzlinie abgelehnt. Das möchte ich hier einmal feststellen.

(Händeklatschen in der Mitte und rechts.)

Es ist eine Provokation der überwältigenden Mehrheit dieses Hauses, wenn Sie derartige $\rightarrow$ Ausführungen machen. Ich rufe Sie deshalb zur Ordnung!

Aus heutiger Sicht kann die Reaktion auf die Äußerung Max Reimanns, die Oder-Neiße-Linie als „Grenze des Friedens“ und damit als Westgrenze Polens zu betrachten, nur erstaunen. Im Jahr 1949 reicht offensichtlich ein im Parlament vorgebrachter Gedanke dieser Art aus, um tumultartige Reaktionen und aggressive Beschimpfungen auszulösen. Doch damit nicht genug: Der Redner erhält für seine Ausführungen vom Bundestagspräsidenten, Erich Köhler (CDU), sogar einen Ordnungsruf. Das wirft nicht nur ein bezeichnendes Licht auf den Spielraum freier Meinungsäußerungen in der Adenauer-Ära, sondern zeigt ebenso, wie verhärtet gewisse Positionen in der Zeit des Kalten Krieges doch sind (vgl. auch Abb. 1).

Dabei hatten bereits die Beschlüsse der Konferenz von Jalta im Februar 1945 deutlich gemacht, eine Westverschiebung der Grenzen Polens vorzusehen, wenn auch noch unter dem Vorbehalt einer friedensvertraglichen Regelung; außerdem bestand noch keine Einigung bezüglich des Grenzverlaufs. Die Potsdamer Konferenz (Juli-August 1945) bestätigt die zuvor getroffenen Entscheidungen, und die rasch eingesetzte Umsiedlungspolitik sorgt

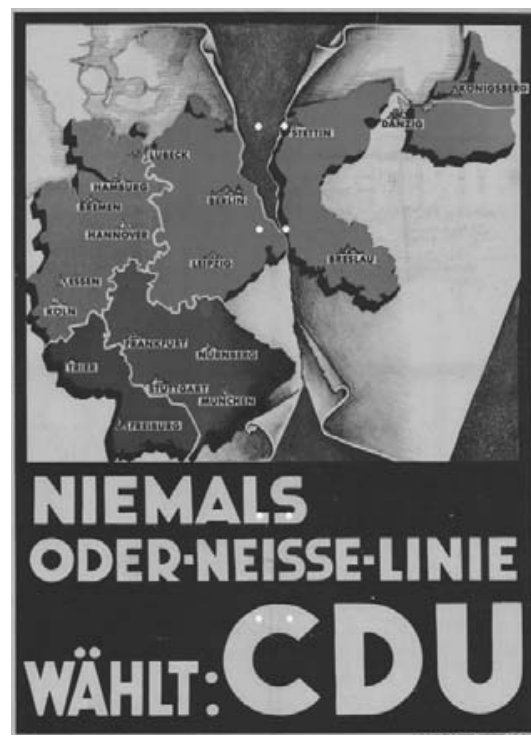

Abb. 1: Wahlplakat der CDU (Landtagswahl Nordrhein-Westfalen 1947) gleichsam für eine Unumkehrbarkeit der Entwicklung. Auf jeden Fall ist 1949 allen Mitgliedern des deutschen Bundestags diese Lage präsent, alle Diskussionsteilnehmer wissen um die faktische Unmöglichkeit einer neuerlichen (und friedlichen) Grenzrevision in Richtung Osten. Die im obigen Ausschnitt (1) vorgeführte

6 So stimmen die beiden Westalliierten Großbritannien und die USA erst spät der Lausitzer Neiße als vorläufiger Grenze Polens (anstelle der weiter südöstlich verlaufenden Glatzer Neiße) zu. 
Auseinandersetzung ist insofern eher eine Scheindiskussion, die gezeigten Emotionen sind vor allem Teil parteipolitischer Abgrenzungs- und Diskreditierungsbemühungen. Gegenüber der Öffentlichkeit handelt es sich um das Wachhalten von Hoffnungen, deren Einlösung angesichts der gegebenen Machtverhältnisse und der erfolgten Eingliederung der Gebiete östlich der Oder-Neiße-Linie in den polnischen Staat zunehmend unrealistisch erscheinen.

Bezogen auf das Jahr 1970 ist zwar einige Zeit vergangen, und die nach dem Krieg etablierten oder durchgesetzten Verhältnisse haben sich weitgehend stabilisiert. Dies schließt

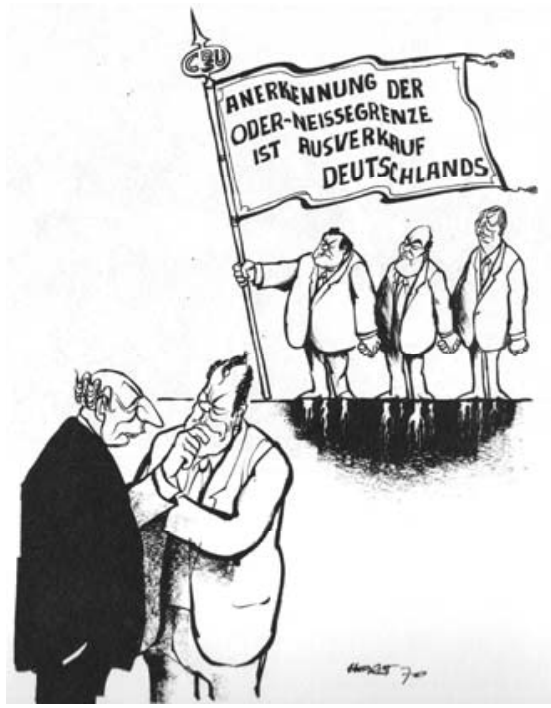

„Einmal müssen sie es ja erfahren, wir werden es ihnen also schonend beibringen, daß wir 1945 den Krieg verloren haben!“

Abb. 2: Oder-Neiße-Linie als politisches Streitobjekt (Horst Haitzinger 1970, aus: Dollinger / Keim 1989: 90) jedoch ein Beharren auf bestimmten Positionen, z. B. der Vorläufigkeit der Oder-Neiße-Linie, nicht aus, und zwar unabhängig davon, welche „Umstände“ und Maßnahmen eine grundsätzliche Neuregelung konkret erfordern würde. Aus diesem Grunde stoßen Verschiebungen bezüglich der außenpolitischen Orientierung von vornherein auf großen Widerstand, und gerade die Ablehnung der Oder-Neiße-Linie als Westgrenze Polens gilt vielen deutschen Politikern als unumstößlich und nicht diskutierbar. Die Karikatur in Abb. 2 veranschaulicht die Gegensätzlichkeit der Haltung von Bundesregierung und Opposition: Die Vertreter der Christdemokraten (Franz Josef Strauß, Rainer Barzel, Kurt Georg Kiesinger) und die sozialliberale Koalition (Willy Brandt, Walter Scheel) stehen sich unversöhnlich gegenüber.

Mit der Einleitung ihrer neuen Ostpolitik sieht sich die Bundesregierung also mit massiven Vorbehalten konfrontiert. Die erste Etappen bilden die Vereinbarungen des Moskauer Vertrags vom 12. August 1970, gefolgt vom Warschauer Vertrag am 7. Dezember 1970; weitere Verträge schließen sich an, so mit der DDR (1971) und der Tschechoslowakei (1973). Im Zusammenhang mit der Ratifizierung dieser Vereinbarungen kommen allerdings die Einwände der Opposition, die bekanntlich den Kurswechsel radikal ablehnt, zum Tragen: Moniert werden das einseitige Gewähren von Vorleistungen und die weitgehende Preisgabe deutscher Interessen. Zentraler Stein des Anstoßes ist jedoch, wie die Opposition unterstellt, die De-facto-Anerkennung der Oder-Neiße-Linie als Westgrenze Polens.?

Die skizzierten Positionsunterschiede kommen ausführlich und uneingeschränkt in den Bundestagsdebatten zum Ausdruck. Ein Einlenken der Opposition gibt es erst, als der

7 Eine endgültige und völkerrechtlich verbindliche Vereinbarung zur Grenze zwischen Deutschland und Polen stellt - im Gefolge der deutschen Einigung - schließlich 1990 der Deutsch-Polnische Grenzvertrag dar. - Zur Vermittlungsproblematik und zur begrenzten Bereitschaft in der bundesdeutschen Öffentlichkeit, die veränderten politischen Realitäten wahrzunehmen, vgl. ausführlicher NIEDHART (2019). 
Versuch, die Regierung zu übernehmen, im April 1972 fehlschlägt und nur noch ein kleiner Teil der christdemokratischen Opposition die Ratifizierung der Verträge zu verhindern sucht. Schließlich sorgen die vorgezogenen Neuwahlen zum Deutschen Bundestag Ende 1972 für eine Bestätigung der sozialliberalen Koalition (sie erhält 54\% der Stimmen), damit ist eine Fortsetzung des eingeschlagenen ostpolitischen Kurses möglich.

\section{Kontroversen um den Warschauer Vertrag von 1970}

Da die parlamentarische Auseinandersetzung in diesem Rahmen nicht in allen Details dokumentiert werden kann, beschränken sich die folgenden Abschnitte vor allem auf die Besprechung zweier Texte: a) der Fernsehansprache des Bundeskanzlers Willy Brandt vom 7. Dezember 1970, b) der Rede des Oppositionsführers Rainer Barzel vom 28. Januar 1971.

\subsection{Proponenten-Sicht}

Unmittelbar nach der Unterzeichnung des Warschauer Vertrags wendet sich der Bundeskanzler in einer Fernsehansprache direkt an die deutsche Bevölkerung. Die Rede ist gekennzeichnet von dem Bemühen, die mit dem Vertrag verbundenen Folgen als positiv darzustellen, andererseits die vorhersehbaren Einwände und Widerstände anzusprechen und gleichsam im Vorhinein zu beschwichtigen. Dementsprechend finden sich bereits in den ersten Abschnitten verschiedene konstruktiv klingende, akzeptanzwerbende Ausdrücke, die die Brisanz des ostpolitischen Kurswechsels andeuten:

(2a) Meine lieben Mitbürgerinnen und Mitbürger!

Ich bin mir bewußt: Dies ist eine schwere Reise. Für eine friedliche Zukunft wird sie von Bedeutung sein. Der Vertrag von Warschau soll einen Schlußstrich setzen unter Leiden und Opfer einer bösen Vergangenheit. Er soll eine Brücke schlagen zwischen den beiden Staaten und den beiden Völkern. Er soll den Weg dafür öffnen, dass getrennte Familien wieder zusammenfinden können. Und dass Grenzen weniger trennen als bisher. [...]

Nichts ist heute wichtiger als die Herstellung eines gesicherten Friedens. Dazu gibt es keine Alternative. Frieden ist nicht möglich ohne europäische Solidarität. Alles, was uns diesem Ziel näher bringt, ist ein guter Dienst an unserem Volk und vor allem ein Dienst für die, die nach uns kommen. [...] (Fernsehansprache von Bundeskanzler Willy Brandt, Warschau, 7.12.1970; Hervorhebungen H.H.L.) ${ }^{8}$

Ausdrücke wie friedliche Zukunft, eine Brücke schlagen, gesicherter Frieden bestätigen eine affektgeladene Wortschatzwahl, der man, um es mit Dieckmann (1969: 76) zu formulieren, sowohl eine emotive wie auch appellative Funktion zuschreiben kann. Brandt ist sich ganz offensichtlich sehr darüber im Klaren, mit welchen Vorbehalten er bei der Bevölkerung, der parlamentarischen Opposition und vor allem bei den Vertriebenenverbänden zu rechnen hat.

8 Vgl. auch LÜGER (2017); der vollständige Redetext ist einsehbar unter: https://www.willy-brandt.de/ fileadmin/brandt/Downloads/Fernsehansprache_Willy_Brandt_Warschau_1970.pdf (21.11.2018). 
Von daher bemüht er Ausdrücke und Wendungen, die, für sich genommen, nur positive und in die Zukunft gerichtete Reaktionen stimulieren sollen; es handelt sich um die Äußerung von Bewertungshandlungen, verbunden mit der Hoffnung, auf eine gewisse Bereitschaft zur Übernahme zu stoßen. Über die tatsächlich ausgelösten Konsequenzen auf Adressaten-Seite ist damit jedoch noch nichts gesagt.

Der Bundeskanzler begnügt sich jedoch nicht mit selbstlobenden Äußerungen. Ebenso werden vorweggenommene Einwände in die Rede einbezogen; sie fungieren, wie oben angedeutet, als Beschwichtigung antizipierter Vorbehalte und zielen auf eine Schwächung ablehnender Positionen ab:

(2b)

\begin{tabular}{|c|c|}
\hline \multicolumn{2}{|c|}{ WARSCHAUER VERTRAG } \\
\hline ZIELE & BESCHWICHTIGUNGEN \\
\hline $\begin{array}{l}\text { - friedliche Zukunft, Erreichen europäi- } \\
\text { scher Friedensordnung } \\
\text { - Schlußstrich unter Leiden und Opfer } \\
\text { einer bösen Vergangenheit, Aussöh- } \\
\text { nung } \\
\text { - Brücke zwischen den beiden Staaten } \\
\text { und den beiden Völkern } \\
\text { - Erkennen der Wirklichkeit } \\
\text { - Politik der Vernunft } \\
\text { - Zusammenfinden getrennter Familien }\end{array}$ & $\begin{array}{l}\text { - ... gibt nichts preis, was nicht längst } \\
\text { verspielt worden ist } \\
\text { - kein Anerkennen von Unrecht oder } \\
\text { Gewalttaten } \\
\text { - kein nachtägliches Legitimieren von } \\
\text { Vertreibungen } \\
\text { - keine Politik des Verzichts } \\
\text { - kein formeller Friedensvertrag } \\
\text { - Verantwortlichkeiten der Vier Mächte } \\
\text { nicht berührt } \\
\text { - Mitwirkung in den westeuropäischen } \\
\text { Gemeinschaften weiterhin Grundlage }\end{array}$ \\
\hline
\end{tabular}

Die Rede hebt die Notwendigkeit hervor, endlich mit der Vergangenheit abzuschließen und den Weg zur Aussöhnung freizumachen, ohne dabei irgendeine Form der Anerkennung auszusprechen, vor allem aber keinem Friedensvertrag vorzugreifen. Der Warschauer Vertrag diene als völkerverbindende Brücke, eine Legitimation von Vertreibungen sei damit ebenso wenig verbunden wie eine Politik des Verzichts - es werde nichts aufgegeben, was "nicht längst verspielt worden" sei. Die europäische Friedensordnung gebiete eine Politik der Vernunft; dies schließe zum einen die Aufgabe unerfüllbarer Ansprüche ein, zum andern auch den „Mut, die Wirklichkeit zu erkennen“. All das berühre weder den Status der Vier Mächte noch die Verankerung der Bundesrepublik im Atlantischen Bündnis.

Der Aufbau der Fernsehansprache weist also eine klare argumentative Struktur auf. Das Werben um Zustimmung für den Warschauer Vertrag erfolgt mittels einer zweigleisigen Vorgehensweise: Den akzeptanzstützenden Argumenten des Proponenten stehen verschiedene Opponenten-Argumente entgegen, die zwar aufgegriffen, aber sogleich aus Proponenten-Sicht widerlegt oder zumindest entschärft werden. Zur Veranschaulichung der argumentativen Umsetzung sei noch eine zentrale Passage des Redetexts zitiert: 
(2c) [...] [1] Dabei muß man von dem ausgehen, was ist; was geworden ist, auch in Bezug auf die Westgrenze Polens. Niemand hat uns zu dieser Einsicht gezwungen. Wir sind mündig geworden. Es geht um den Beweis unserer Reife und um den Mut, die Wirklichkeit zu erkennen.

[2] Was ich im August Ihnen aus Moskau gesagt habe, liebe Mitbürgerinnen und Mitbürger,

$\rightarrow$ gilt auch für den Vertrag mit Polen: Er gibt nichts preis, was nicht längst verspielt worden ist. Verspielt nicht von uns, die wir in der Bundesrepublik Deutschland politische Verantwortung tragen und getragen haben. Sondern verspielt von einem verbrecherischen Regime, vom Nationalsozialismus.

$\rightarrow \quad$ [3] Wir dürfen nicht vergessen, dass dem polnischen Volk nach 1939 das Schlimmste zugefügt wurde, was es in seiner Geschichte hat durchmachen müssen. Dieses Unrecht ist nicht ohne Folgen geblieben.

$\rightarrow \quad$ [4] Großes Leid traf auch unser Volk, vor allem unsere ostdeutschen Landsleute. Wir müssen gerecht sein: Das schwerste Opfer haben jene gebracht, deren Väter, Söhne oder Brüder ihr Leben verloren haben. Aber nach ihnen hat am bittersten für den Krieg bezahlt, wer seine Heimat verlassen mußte. Ich lehne Legenden ab, deutsche wie polnische. Die Geschichte des deutschen Ostens läßt sich nicht willkürlich umschreiben. [...]

(Hervorhebungen H.H.L.)

Als zentrale Texthandlung, als Hauptthese kann man in (2c) [1] die Forderung ansehen, man müsse endlich die politische Situation in ihrer Realität sehen, nämlich als das, „was ist, was geworden ist“; in diese Forderung schließt Brandt ausdrücklich die „Westgrenze Polens“ mit ein. Die Bezeichnung Oder-Neiße-Linie wird dabei konsequent vermieden, dies vermutlich deshalb, weil der Ausdruck seit Jahren fest mit ablehnenden Slogans („Oder-Neiße - niemals Grenze!“”Die Anerkennung der Oder-Neiße-Linie ist Verbrechen an Deutschland“; vgl. auch Abb. 1) verknüpft ist. ${ }^{9}$

Zur Akzeptanzsicherung der eingangs formulierten These werden anschließend einige Argumente angeführt und mit ergänzenden Erklärungen versehen. Die Behauptung, es werde nichts preisgegeben (in [2]), fungiert als erster, eventuell sogar wichtigster Stützungsversuch: Der zusätzlich begründende Rekurs auf die Verantwortlichkeit des nationalsozialistischen Regimes appelliert gleichsam an einen parteiübergreifenden Konsens und soll ebenfalls die These legitimieren helfen. Das zweite Argument bringt die an der polnischen Bevölkerung im Zweiten Weltkrieg begangenen Verbrechen zur Sprache (in [3]); die Respektierung der Westgrenze Polens ist, so gesehen, also auch eine notwendige Konsequenz aus der jüngsten Geschichte. Weniger eindeutig erscheint dagegen der argumentative Status von Abschnitt [4]. Bekanntlich stellen die Vertriebenen eine starke, gut organisierte und äußerst öffentlichkeitswirksame Oppositionsgruppe dar; sie beklagen u. a., mit ihrer Forderung eines Rückkehrrechts in die nun zu Polen gehörenden Gebiete jenseits der Oder-Neiße-Linie bei der aktuellen Regierung kein Gehör zu finden. Hierauf reagiert Brandt in seiner Ansprache insofern, als er in einer solidarisch klingenden Äußerung die großen Opfer des eigenen Volkes hervorhebt und speziell auch die Menschen erwähnt, die zum Verlassen ihrer Heimat gezwungen waren. Mit dieser auch pronominal markierten Solidarisierungsgeste (wir, zweimaliges

9 Zu bedenken wäre möglicherweise auch der Sprachgebrauch der DDR: Seit dem Görlitzer Abkommen von 1950 wird die Oder-Neiße-Linie offiziell als „Friedens- und Freundschaftsgrenze“ bezeichnet. 
unser) geht jedoch kein politisches Zugeständnis einher. Von daher kann man [4] als indirekte Zurückweisung einer Opponenten-Forderung und in der Konsequenz ebenso als weitere Absicherung der übergeordneten These auffassen - Geschichte lasse sich „nicht willkürlich umschreiben“. Zur Verdeutlichung sei die Stringenz des argumentativen Vorgehens noch einmal schematisch festgehalten:

$\left(2 c^{\prime}\right)$

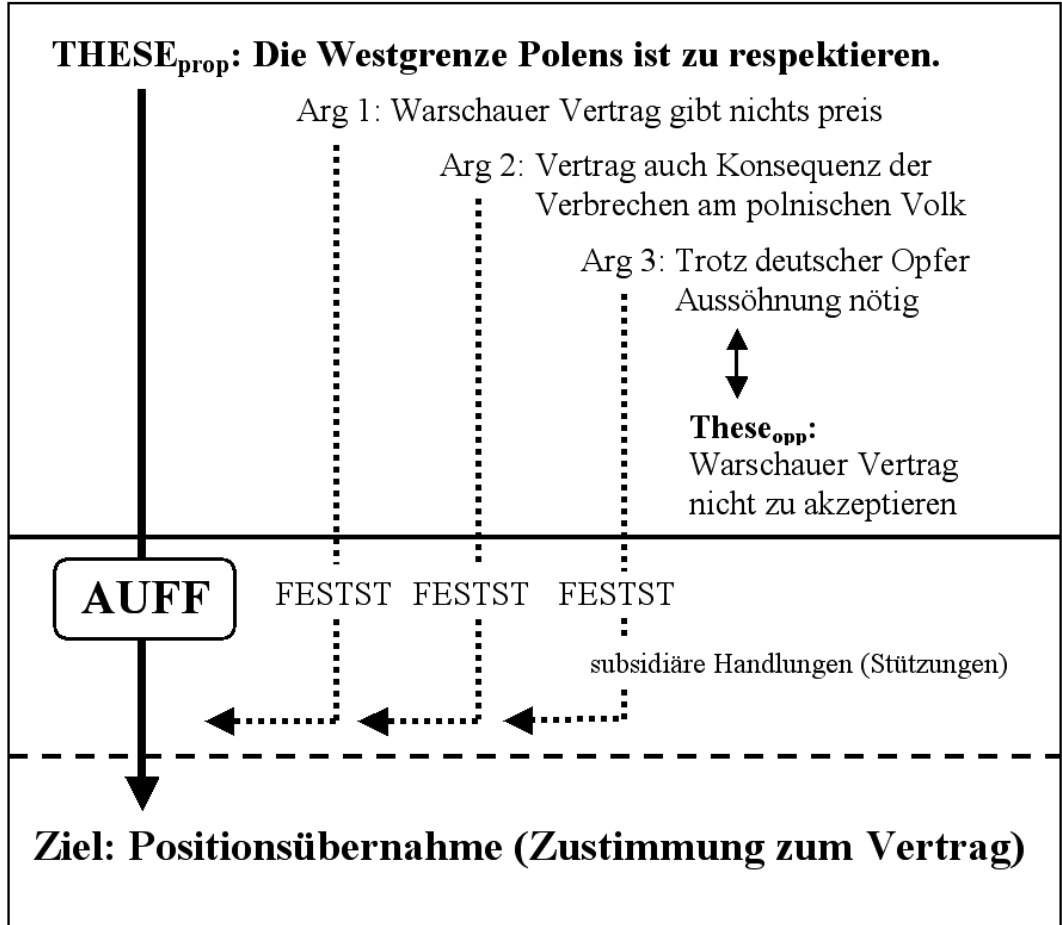

Der investierte sprachlich-argumentative Aufwand bedeutet jedoch noch keine Erfolgsgarantie; die zentrale Aufforderung braucht also nicht die intendierte Zielreaktion zur Folge zu haben. Im Gegenteil, wie die politische Auseinandersetzung um die Ostpolitik und insbesondere um den Warschauer Vertrag zeigt, ist bereits die Akzeptanz der einzelnen Argumente umstritten: So wird die Feststellung Brandts, der Vertrag gebe nichts preis, von der Gegenseite vehement bestritten (vgl. 3.2); auch die Konsequenzen, die man aus den deutschen Kriegsverbrechen in Polen ziehen müsse, werden unterschiedlich beurteilt oder im gegebenen Zusammenhang gar nicht erst thematisiert; und schließlich kommt den Einlassungen der Vertriebenenverbände bei der Opposition ein ganz anders Gewicht zu..$^{10}$ Damit entfällt dann letztlich auch eine allgemeine Grundlage für die Zustimmung zur Hauptthese der Regierung, die Westgrenze Polens sei als solche nicht länger in Frage zu stellen.

10 Als folgenschwer für die Mehrheitsverhältnisse im Deutschen Bundestag erweisen sich ebenso die Reaktionen einiger Abgeordneter, die die Regierungskoalition aus Protest gegen die Ostpolitik verlassen und zur CDU/CSU-Opposition übertreten, ohne jedoch ihr Mandat, für das sie gewählt wurden, zur Verfügung zu stellen. Auf diese Weise verliert die Brandt-Scheel-Regierung bis 1972 im Parlament ihre Mehrheit. 


\subsection{Opponenten-Sicht}

Die CDU/CSU-Opposition im Deutschen Bundestag nutzt jede sich bietende Gelegenheit, der Regierung Fehler, Versäumnisse und fundamentale Fehleinschätzungen der politischen Kräfteverhältnisse vorzuhalten. Ein Beispiel (unter vielen) für diese Haltung ist die Kritik, wie sie der Sprecher der CDU/CSU, Rainer Barzel, im Rahmen der „Aussprache über den Bericht zur Lage der Nation 1971 " vorträgt. ${ }^{11}$ Aus dieser Rede seien einige Ausschnitte zitiert:

(3a) [...] Herr Bundeskanzler, von den notwendigen innerdeutschen Regelungen, von Entspannung in Deutschland - so sprachen Sie z. B. in Erfurt davon, daß alle Deutschen von dieser Politik etwas haben müssen -, von alledem reden Sie nun nur noch am Rande. Für uns ist das der Kern einer vernünftigen Ostpolitik,

(Beifall bei der CDU/CSU)

$\rightarrow$ und ohne Lösungen im Kern, der Lage der Deutschen in Deutschland, und ohne unwiderrufliche Fortschritte in diesem Zentralpunkt werden Sie, Herr Bundeskanzler, zwar versuchen können, das von Ihnen geschnürte Gesamtpaket aufzuschnüren - dies war doch Ihre Ankündigung von heute -, aber dabei werden wir Ihnen freilich nicht helfen, sondern wir werden die Ratifikation - jetzt gebrauche ich ein Wort Ihres Herrn Außenministers - von „Fragmenten“ in diesem Hause zu bekämpfen wissen.

(Beifall bei der CDU/CSU) (Hervorhebungen H.H.L.)

Während sich die Bundesregierung ganz auf außenpolitische Belange konzentriert, stellt die Opposition Innerdeutsches in den Mittelpunkt: die „Lage der Deutschen in Deutschland“; hier liege, so Barzel, der „Kern einer vernünftigen Ostpolitik“. Gleichzeitig wird deutlich, mit welcher Vehemenz die Opposition den neuen außenpolitischen Kurs zu bekämpfen gedenkt. Die Gründe für die Selbstsicherheit, mit der Barzel seine Absicht bekundet, die Ratifikation des Vertrags erschweren oder verhindern zu wollen, mögen durchaus von der knappen und unsicheren parlamentarischen Mehrheit der Regierungskoalition herrühren.

Mit Bezug auf die Unterzeichnung des Warschauer Vertrags richtet sich die Argumentation vor allem gegen die praktizierte Vorgehensweise und die aus Sicht der Opposition damit verbundene Preisgabe wichtiger deutschlandpolitischer Positionen. Zusammenfassend wird der Vorwurf eines „Programms der Vorleistungen“ erhoben, ein Vorwurf, der dann noch eine weitere Spezifizierung erfährt; genannt werden in dem Zusammenhang folgende Argumente: - Behauptet wird, nach dem Wortlaut des Vertrags spreche die Regierung (in Artikel I) eine endgültige Anerkennung der Oder-Neiße-Linie aus. ${ }^{12}$

11 Deutscher Bundestag, 6. Wahlperiode, 93. Sitzung, 28.1.1971, S. 5051-5058; Redeprotokoll einsehbar unter: http://dipbt.bundestag.de/doc/btp/06/06093.pdf (5.6.2019).

12 Hierzu ist Folgendes anzumerken: Der Ausdruck Anerkennung wird in allen offiziellen Dokumenten der Regierung konsequent vermieden, und zwar wegen des Vorbehalts der Vier Mächte für Deutschland als Ganzes. Im Warschauer Vertrag heißt es unter Artikel I:

„(1) Die Bundesrepublik Deutschland und die Volksrepublik Polen stellen übereinstimmend fest, daß die bestehende Grenzlinie, deren Verlauf im Kapitel IX der Beschlüsse der Potsdamer Konferenz vom 2. August 1945 von der Ostsee unmittelbar westlich von Swinemünde und von dort die Oder entlang bis zur Einmündung der Lausitzer Neiße und die Lausitzer Neiße entlang bis zur Grenze mit der Tschechoslowakei festgelegt worden ist, die westliche Staatsgrenze der Volksrepublik Polen bildet. (2) Sie bekräftigen die Unverletzlichkeit 
- Die Verträge von Moskau und Warschau würden eine Aufwertung der DDR bewirken. Diese Behauptung wird noch mit einer zusätzlichen Erklärung in Sachen DDR untermauert.

- Die Bundesregierung habe eine parteienübergreifende Basis des Deutschen Bundestages - gemeint ist wohl die gemeinsame Resolution vom September 1968 - verlassen.

- Ebenso seien die Verbündeten auf westalliierter Seite vor vollendete Tatsachen gestellt worden (vgl. die resümierende Darstellung in (3b)).

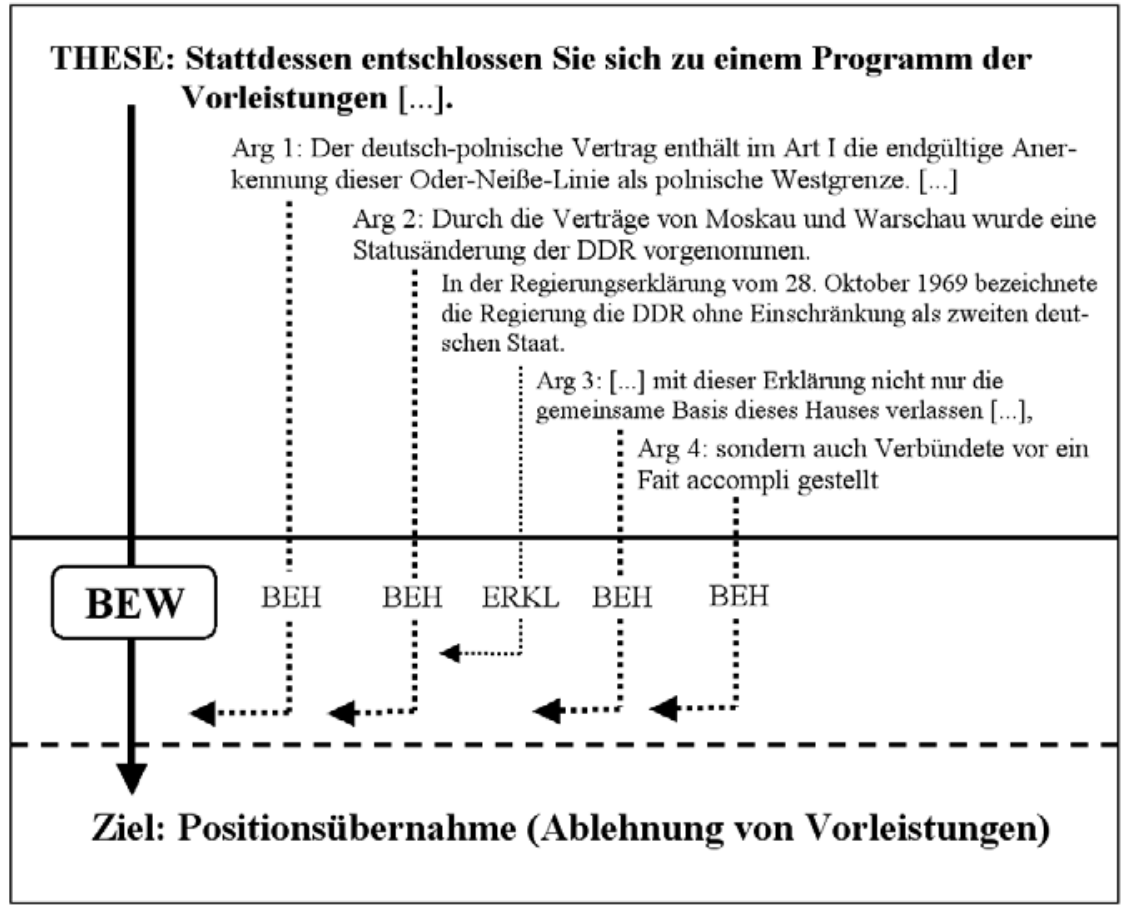

Der Vorwurf der Vorleistungen zieht sich als Negativbewertung wie ein roter Faden durch die gesamte Rede Barzels. Bei den dabei herangezogenen Argumenten werden gelegentlich auch eher fragwürdig anmutende Behauptungen bemüht; so hat es z. B. die unterstellte „endgültige Anerkennung“ der Westgrenze Polens nicht gegeben, oder die Aussage, die Verbündeten wären vor vollendete Tatsachen gestellt worden, ist in der Form (wegen der zuvor stattgefundenen Konsultationen) nicht haltbar. Der Abschnitt (3b) fungiert mit seiner Hauptthese innerhalb des Redetexts als wichtiger argumentativer Block, der wiederum die zentrale Texthandlung stützen soll, nämlich die Forderung, vom eingeschlagenen ostpolitischen Kurs

ihrer bestehenden Grenzen jetzt und in der Zukunft und verpflichten sich gegenseitig zur uneingeschränkten Achtung ihrer territorialen Integrität. (3) Sie erklären, daß sie gegeneinander keinerlei Gebietsansprüche haben und solche auch in Zukunft nicht erheben werden.“ (zitiert nach: http://www.documentarchiv.de/brd/1970/ warschauer-vertrag.html; 6.6.2019). 
abzurücken. Diese Handlung kommt mehrfach und zum Teil mit sehr deutlichen Worten zum Ausdruck:

(3c) [...] Herr Bundeskanzler, wir fordern Sie auf: Keine Vorleistungen mehr! [...] Solange Ost-Berlin den Kalten Krieg anheizt, keine Erleichterungen gewährt und Entspannung nicht will, darf diese Bundesregierung keinen Millimeter weitergehen [...]. (Hervorhebungen im Original)

(3d) Meine Damen und Herren, wir halten daran fest - entgegen dem, was der Bundeskanzler gesagt hat -, daß wir das Ergebnis dieser Ostpolitik in s ge s a m t zu würdigen wünschen, daß „Fragmente", die vorher vorgelegt werden, von vornherein nicht mit unserer Unterstützung rechnen können. Damit wir uns ganz verstehen, füge ich hinzu: Die Verträge und Ergebnisse, die uns bisher vorliegen, entsprechen den Maßstäben, die ich eben nannte, nicht. Die Deutschen in Deutschland haben nämlich von dieser Politik bisher nichts; sonst hätte der Bundeskanzler wenigstens e i n e n Punkt vorgetragen. (Hervorhebungen im Original)

Die argumentative Makrostruktur der Rede des Oppositionsführers sei noch einmal schematisch veranschaulicht:

(3’)

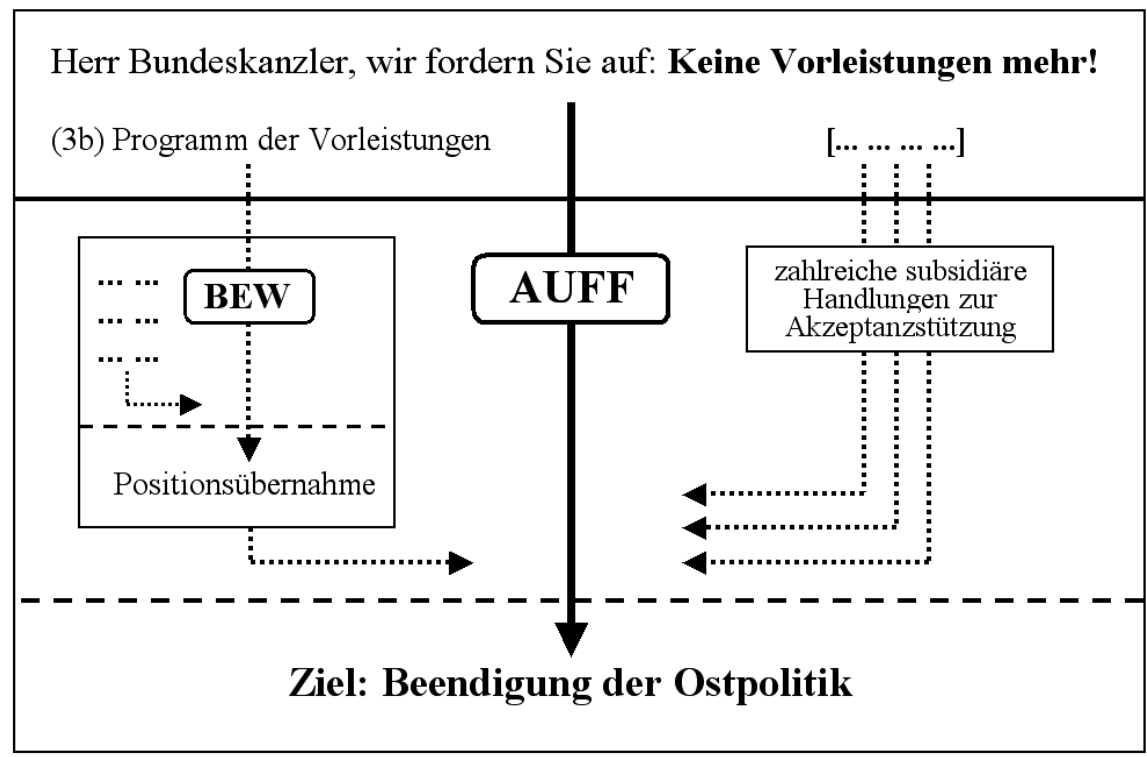

Nicht berücksichtigt ist in den Schaubildern bisher der Faktor ,Mehrfachadressiertheit': Bei der Zuschreibung von Sprachhandlungen steht jeweils die primäre Adressatengruppe, die Abgeordneten und die Anhänger der eigenen Partei, im Vordergrund. Aus der Sicht anderer Adressatengruppen kommen zwangsläufig auch andere Äußerungsinterpretationen in den Blick. Gleiches gilt für die zugeordneten Ziele bzw. die erwarteten Reaktionen auf Adressatenseite. 


\section{Realitätsflucht und Feindbildkommunikation}

Zu Beginn der 1970er Jahre scheint die Bereitschaft zu einer Kursänderung bezüglich der Ost-West-Beziehungen noch höchst unterschiedlich ausgeprägt zu sein. Begriffe wie „Realitätsbereitschaft“ und „Realitätsflucht“ kennzeichnen die Situation. Die skizzierte parlamentarische Auseinandersetzung liefert hierfür sprechende Beispiele.

„Die Kontroverse über die Wahrnehmung der Realität begleitete jede ostpolitische Initiative. Nur langsam vollzog sich ein Perspektivenwechsel, bei dem die Nachkriegsrealität nicht mehr überwiegend als verlustreiche Gewalterfahrung wahrgenommen wurde, sondern zunehmend auch als Ausgangspunkt für einen ,Dialog auf neuer Ebene““' (NIEDHART 2019: 45f.)

Die inhaltlichen Schwerpunkte in der Rede Barzels lassen allerdings einen solchen Perspektivenwechsel noch nicht erkennen. Es dominieren in erster Linie Themen, die deutschlandbezogen sind: Situation an der „Zonengrenze“, Rückgang der Reisen in die DDR, einseitiger „Interzonenhandel“, strittiger Status von Berlin, Behinderungen des Personenverkehrs nach Berlin, Abnahme des innerdeutschen Sportverkehrs, Ausreisemöglichkeiten für Deutsche aus Polen. Damit einher geht eine polarisierende Schärfung des hinlänglich bekannten politischen Feindbilds:

(3e) Wir, meine Damen und Herren, sind immer von der sehr weitgehenden Übereinstimmung der Interessen zwischen der SED und der KPdSU und zwischen der DDR und der Sowjetunion ausgegangen. Ist es nicht vielleicht eine sowjetische Taktik, daß man Moskau den besseren Willen unterstellt und Ulbricht ganz bewußt den Part des „Bremsers“, des „Quertreibers“, des „Querulanten“ spielen läßt? Ich meine, keiner von uns sollte sich seine europäische Szenerie nach Wunschbildern zurechtmachen.

Angesichts eines so prononciert vorgetragenen Feindbilds wird plausibel, warum es 1971 noch zu keinem „Dialog auf neuer Ebene“ kommen kann. Wie verhärtet die Positionen im Deutschen Bundestag letztlich sind, demonstriert auch die ein Jahr später gehaltene Rede Rainer Barzels, in der es wieder um die Ostpolitik und die vermeintlichen „Vorleistungen“ geht: ${ }^{13}$

(4) Warum rühmen sich die Führer des Ostblocks nun dieser Verträge als ihrer großen Siege? Doch wohl nicht, weil sie mit diesen Verträgen nichts bekommen haben. [...] Wer die deutschen Vorleistungen dieses Vertrages als „nichts“ bezeichnet, hat zu Aussöhnung und Geschichte ein anderes Verhältnis als wir! [...]

Sie sagen, Ihre Politik und diese Verträge machten „den Frieden sicherer“. Wer wollte, wenn das real und solide mit Tatsachen begründet wäre, dem die Zustimmung versagen? Doch keiner! Aber wo sind die Tatsachen, die diese Aussage begründen? Sie argumentieren mit Hoffnungen, wo Tatsachen, und mit Perspektiven, wo Wirklichkeiten entscheidend sind. Die anderen bekommen konkret, was sie wollen: Tatsachen. Wir werden mit Hoffnungen abgespeist.

(Hervorhebungen im Original)

13 Deutscher Bundestag, 6. Wahlperiode, 171. Sitzung, 23.2.1972, S. 9763; Redeprotokoll einsehbar unter: http://dip21.bundestag.de/dip21/btp/06/06171.pdf(5.6.2019). 
Die Politik der sozialliberalen Koalition wird als wirklichkeitsfremd, als von „Wunschbildern“, „Perspektiven“ und „Hoffnungen“ geleitet, charakterisiert. Und geradezu leitmotivisch kommen „Verzicht" und „deutsche Vorleistungen“" zur Sprache. Ausgeklammert bleiben dagegen Themen wie die deutschen Kriegsverbrechen in Polen oder eine Diskussion des Hauptziels der neuen Ostpolitik, der Aussöhnung mit den Völkern Osteuropas.

Auch sprachlich herrscht wenig Zurückhaltung, wenn es um die Kritik an der Ostpolitik geht. Um die politische Neuausrichtung zu diskreditieren, werden immer wieder negativ besetzte Bezeichnungen wie „Verzicht dieser Bundesregierung "oder „deutsche Vorleistungen“ ins Spiel gebracht. In die gleiche Richtung geht die ständig wiederholte Redeweise von einer „Anerkennung der Oder-Neiße-Linie“. Damit wird der deutschen Bevölkerung, und die ist hier als eigentlicher Adressat der Reden zu sehen, suggeriert, die Brandt-Scheel-Regierung übergehe den juristischen Vier-Mächte-Vorbehalt und betreibe eine Preisgabe deutscher Interessen. Umgekehrt kommen zur Aufwertung der eigenen Position Wörter und Formeln mit großem positiven Emotionswert zum Einsatz, z. B. „Selbstbestimmungsrecht“, „Grundfreiheiten und Grundrechte“, „innerdeutscher Fortschritt“, „Friedenssicherung“, „vernünftige Ostpolitik“, um nur einige Beispiele zu nennen. Die Selbstdarstellung der CDU/CSU als alleiniger Bewahrer demokratischer Werte ist omnipräsent. Nicht unwichtig dürfte schließlich die eindeutig polarisierende Zuordnung der politischen Aktivitäten sein: Die Gut-BöseKategorisierungen aus der Zeit des Kalten Krieges sind ungebrochen wirksam und nehmen oft die Form personalisierender Herabwürdigungen an.

Trotz der überaus kontroversen und von Polemik und vielen Feindseligkeiten begleiteten Debatten wird der Warschauer Vertrag am 17. Mai 1972 von der Mehrheit des Deutschen Bundestages (bei überwiegender Stimmenthaltung der CDU/CSU) ratifiziert. Vorausgegangen war der am 27. April 1972 gescheiterte Versuch, die Brandt-Scheel-Regierung zu stürzen; anschließend gibt es für beide Seiten praktisch keine kalkulierbare parlamentarische Mehrheit mehr. Eine wichtige Voraussetzung, die sowohl der Regierung wie auch der Opposition die Gesichtswahrung ermöglicht, ist am Ende die Vereinbarung einer Gemeinsamen Entschließung, in der ausdrücklich die Vier-Mächte-Verantwortung und der Vorbehalt einer friedensvertraglichen Regelung festgehalten werden. Damit ist der Weg frei für die Ratifizierung und das Wirksamwerden des Warschauer Vertrags.

Im Zuge der deutschen Einigung wird am 12. September 1990 der Zwei-plus-Vier-Vertrag („Vertrag über die abschließende Regelung in Bezug auf Deutschland“) zwischen der BRD und der DDR einerseits und den vier Alliierten andererseits unterzeichnet. In der Folge beschließen Deutschland und Polen, wie oben erwähnt, am 14. November 1990 den Deutsch-Polnischen Grenzvertrag, vereinbaren damit die Oder-Neiße-Linie als endgültige Grenze zwischen Deutschland und Polen, bestätigen sich gleichzeitig die Unverletzlichkeit der Grenzen und erklären den Verzicht auf künftige Gebietsansprüche. Diese nun offizielle Anerkennung der Oder-Neiße-Linie als Staatsgrenze mag aus Sicht so mancher Karikaturisten als „Sprung über den eigenen Schatten" gesehen werden, zumal dann, wenn es ein Christdemokrat ist, nämlich Helmut Kohl, der diesen gigantischen Sprung vollzieht. ${ }^{14}$

14 Im Hintergrund sind die Politiker Otto Lambsdorff(FDP), Hans-Jochen Vogel (SPD), Lothar de Maizière (CDU, letzter Ministerpräsident der DDR), Theo Waigel (CSU) erkennbar. 


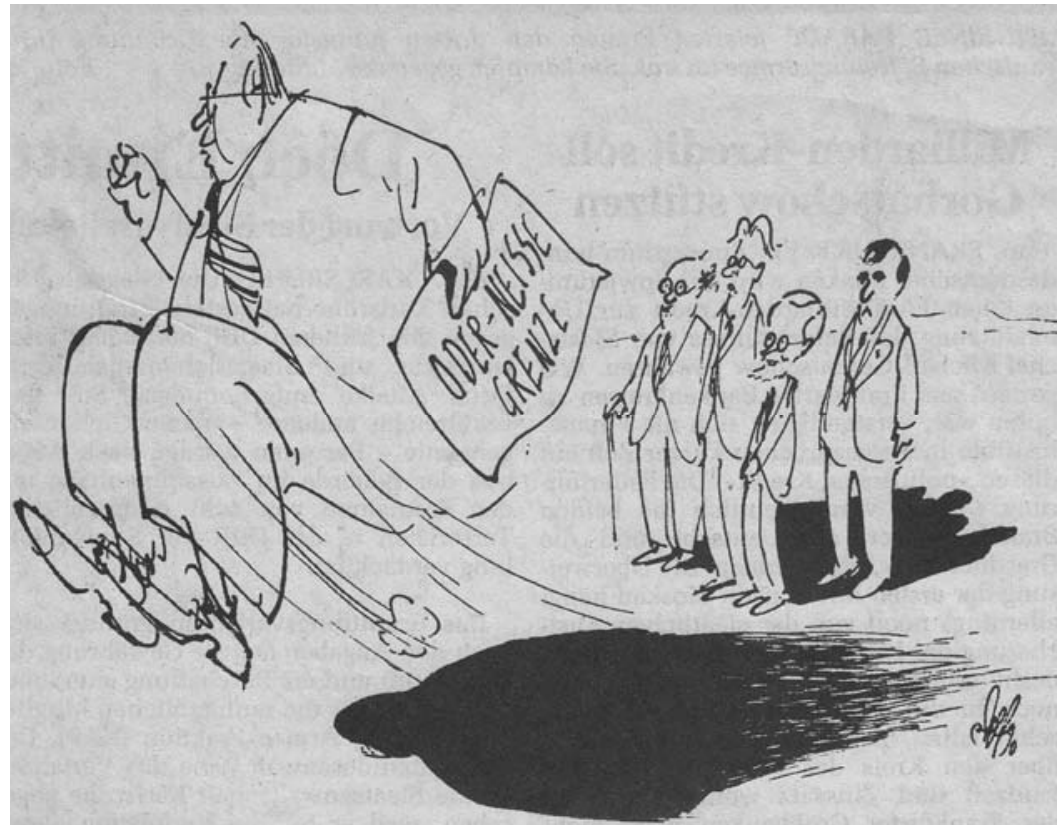

Abb. 3: Sprung über den eigenen Schatten (Fritz Wolf, Schwarzwälder Bote 22.6.1990)

\section{Literatur}

BaCHem, Rolf (1979): Einführung in die Analyse politischer Texte. München.

Chamberlain, Houston Stewart (1914): Kriegsaufsätze. München.

Czachur, Waldemar (2015): Texte machen kollektives Gedächtnis wahrnehmbar! In: OpIŁOwski,

Roman / Czachur, Waldemar (Hg.): Sprache - Wissen - Medien. Festschrift Gerd Antos. Wrocław, Dresden, 55-69.

Dieckmann, Walther (1969): Sprache in der Politik. Einführung in die Pragmatik und Semantik der politischen Sprache. Heidelberg.

Dollinger, Hans / Keim, Walther (Hg.) (1989): Das waren Zeiten... München.

Fetscher, Iring / Richter, Horst E. (Hg.) (1976): Worte machen keine Politik. Beiträge zu einem Kampf um politische Begriffe. Reinbek.

GIRNDT, Heiko (2002): Sprache und Sprachverwendung in der Politik. Tübingen.

Girndt, Heiko / SpIEss, Constanze (Hg.) (2006): Strategien politischer Kommunikation. Pragmatische Analysen. Berlin.

Holly, Werner (1990): Politikersprache. Inszenierungen und Rollenkonflikte im informellen Sprachhandeln eines Bundestagsabgeordneten. Berlin, New York.

Holly, Werner (2012): Sprache und Politik. Pragma- und medienlinguistische Grundlagen und Analysen. Berlin. 
Kaczmarek, Dorota (Hg.) (2016): Politik - Medien - Sprache. Deutsche und polnische Realien aus linguistischer Sicht. Łódź.

Kaltenbrunner, Gerd-Klaus (Hg.) (1975): Sprache und Herrschaft. Die umfunktionierten Wörter. München.

Kienpointner, Manfred (2017): Rhetorik als Vorläufer. In: NieHr, Thomas / Kilian, Jörg / WenGELER, Martin (Hg.): Handbuch Sprache und Politik, Bd. 1. Bremen, 20-32.

Klemperer, Victor (1947): LTI. Notizbuch eines Philologen. Berlin.

Klinker, Fabian / Scharloth, Joachim / SzczęK, Joanna (Hg.) (2018): Sprachliche Gewalt. Formen und Effekte von Pejorisierung, verbaler Aggression und Hassrede. Stuttgart.

LÜGER, Heinz-Helmut (2015): Kommunikation in der Krise. Reden zum Ausbruch des Ersten Weltkrieges. In: WeIGt, Zenon et al. (Hg.): Didaktische und linguistische Implikationen. Łódź, 9-29.

LÜGER, Heinz-Helmut (2017): Phraseologie und politischer Sprachgebrauch. In: BERDYCHOwSKA, Zofia et al. (Hg.): Phraseologie als Schnittstelle von Sprache und Kultur. Bd. II. Frankfurt/M., 35-58.

Makowski, Jacek (Hg.) (2011): How not to do things with words. Beiträge zur Sprache in Politik, Recht und Werbung. Łódź.

Makowski, Jacek (2013): Die Abgeordnetenrede im Europäischen Parlament. Łódź.

Massud, Abdel-Hafiez (2019): Argumentationspraktiken im Vergleich. Landau.

MıкоџAjCzyк, Beata (2004): Sprachliche Mechanismen der Persuasion in der politischen Kommunikation. Frankfurt/M.

Müller, Albrecht (2018): Tödlicher Wandel durch Konfrontation - Was uns vermutlich ins Haus steht. In: BAHR, Adelheid (Hg.): Warum wir Frieden und Freundschaft mit Russland brauchen. Frankfurt/M., 126-132.

Niedhart, G. (2019): Durch den Eisernen Vorhang. Die Ära Brandt und das Ende des Kalten Kriegs. Darmstadt.

Nienr, Thomas (2014): Einführung in die Politolinguistik. Göttingen.

Olszewska, Danuta / KąTny, Andrzej (2012): Vom Text zum Diskurs, genauer gesagt: Vom Text zum Text im Diskurs. In: Studia Germanica Gedanensia 29, 9-22.

REICH, Hans H. (1968): Sprache und Politik. Untersuchungen zu Wortschatz und Wortwabl des offiziellen Sprachgebrauchs in der DDR. München.

SPITZER, Leo (1918): Anti-Chamberlain. Betrachtungen eines Linguisten über Houston Stewart Chamberlains „Kriegsaufsätze“ und die Sprachbewertung im allgemeinen. Leipzig.

Sternberger, Dolf / Storz, Gerhard / Süskind, Wilhelm (1957): Aus dem Wörterbuch des Unmenschen. Hamburg.

Volmert, Johannes (1989): Politikerrede als kommunikatives Handlungsspiel. Ein integriertes Modell zur semantisch-pragmatischen Beschreibung öffentlicher Rede. München. 